\title{
Sweet taste preference in binge-eating disorder: A preliminary investigation
}

\author{
Erica L. Goodman ${ }^{\mathrm{a}, \mathrm{b}}$, Lauren Breithaupt ${ }^{\mathrm{a}, \mathrm{c}, \mathrm{d}}$, Hunna J. Watson ${ }^{\mathrm{a}, \mathrm{e}, \mathrm{f}}$, Christine M. Peat ${ }^{\mathrm{a}, \mathrm{g}}$, \\ Jessica H. Baker ${ }^{\mathrm{a}}$, Cynthia M. Bulik ${ }^{\mathrm{a}, \mathrm{d}, \mathrm{h}}$, Kimberly A. Brownley ${ }^{\mathrm{a}, *}$

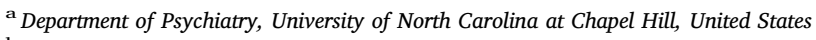 \\ ${ }^{\mathrm{b}}$ Department of Psychology, University of North Dakota, United States \\ ${ }^{\mathrm{c}}$ Department of Psychology, George Mason University, United States \\ d Department of Medical Epidemiology and Biostatistics, Karolinska Institute, Stockholm, Sweden \\ e School of Psychology and Speech Pathology, Curtin University, Perth, Australia \\ ${ }^{\mathrm{f}}$ School of Paediatrics and Child Health, The University of Western Australia, Perth, Australia \\ ${ }^{g}$ Department of Neurosurgery, University of North Carolina at Chapel Hill, United States \\ ${ }^{\mathbf{h}}$ Department of Nutrition, University of North Carolina at Chapel Hill, United States
}

\section{A R T I C L E I N F O}

\section{Keywords:}

Binge-eating disorder

Sweet taste

Food craving

Insulin

Blood glucose levels

\begin{abstract}
A B S T R A C T
Research suggests that individuals with high liking for sweets are at increased risk for binge eating, which has been minimally investigated in individuals with binge-eating disorder (BED). Forty-one adults ( $85 \%$ female, $83 \%$ white) with binge eating concerns completed a sweet taste test and measures of eating disorder behaviors and food cravings. A subset of participants with BED completed an oral glucose tolerance test (OGTT; $N=21$ ) and a 24-hour dietary recall $(N=26)$. Regression models were used to compare highest sweet preferers (HSP $[N=18]$ ) to other sweet preferers (OSP $[N=23]$ ) and were used to assess associations between sweet taste preference and outcome variables. Effect sizes $\left(\eta p^{2}\right)$ for differences between HSP and OSP ranged from small $(\leq 0.01)$ to large $(\geq 0.24)$; group differences were statistically nonsignificant except for 24-hour caloric intake $\left(\eta p^{2}=0.16, p=0.04\right)$, protein intake $\left(\eta p^{2}=0.16, p=0.04\right)$, and insulin sensitivity index $\left(\eta p^{2}=0.24\right.$, $p=0.04)$, which were higher in HSP, and postprandial insulin, which was smaller in HSP $\left(\eta p^{2}=0.27\right.$, $p=0.03$ ). Continuous analyses replicated postprandial insulin response. Compared with OSP, HSP reported numerically higher binge-eating frequency $\left(\eta p^{2}=0.04\right)$, over-eating frequency $\left(\eta p^{2}=0.06\right)$, and carbohydrate intake $\left(\eta p^{2}=0.14\right)$, and they exhibited numerically smaller postprandial glucose AUC $\left(\eta p^{2}=0.16\right)$. Sweet taste preference may have implications for glucose regulation, binge-eating frequency, and nutrient intake in BED.
\end{abstract}

Binge-eating disorder (BED) is characterized by recurrent episodes of binge eating, during which an individual consumes an unusually large amount of food in a short period of time (i.e., about $2 \mathrm{~h}$ ) and experiences a loss of control over eating (American Psychiatric Association [APA], 2000, 2013). Typically, individuals will binge eat on foods high in fats, sugars, or often both (Yanovski et al., 1992). Frequent intake of high calorie and high sucrose foods can lead to increased postprandial glucose and insulin levels, which stimulate hunger (O'Keefe \& Bell, 2007) and may further affect vulnerability to binge eating. Furthermore, binge-eating episodes that are characterized by a high-fat, high-sugar composition may contribute to health risks in BED (Avena, Rada, \& Hoebel, 2009) such as diabetes mellitus, metabolic syndrome, hypertension, psychological distress, impaired glucose tolerance, and other conditions (Hudson et al., 2010; Mitchell et al., 2015; Raevouri et al., 2015; Roehrig, Masheb, White, \& Grilo, 2008; Thornton et al., 2017). Thus, a preference for sweet tasting foods may place someone at greater risk for binge eating and its associated health outcomes; however, little is known about the interactions between sweet liking, craving, and metabolic functions in individuals with BED.

The available literature suggests that preferring sweet tastes may increase the risk for overeating or binge eating in non-eating disorder (ED) populations and in those with BED (Dalton \& Finlayson, 2014; Finlayson, Arlotti, Dalton, King, \& Blundell, 2011; Greeno, Wing, \& Shiffman, 2000; Kampov-Polevoy, Alterman, Khalitov, \& Garbutt, 2006). Sweet preference may impact binge eating in several ways. First, individuals with a strong sweet preference are more likely than individuals with a less strong sweet preference to have difficulty regulating their intake of sweet foods and report consuming sweet foods to decrease depressed mood (Kampov-Polevoy et al., 2006). Thus, individuals with sweet taste preference may be at risk for developing BED as using food intake to regulate negative affect increases risk for overeating (Leibenluft, Fiero, Bartko, Moul, \& Rosenthal, 1993).

\footnotetext{
* Corresponding author at: UNC Center of Excellence for Eating Disorders, CB \#7175, 307 MacNider, UNC Department of Psychiatry, United States. E-mail address: kim_brownley@med.unc.edu (K.A. Brownley).
} 
Second, sweet preference may impact not only the motivation and/or amount one eats, but also what one eats. Several studies have found that individuals who prefer sweet foods tend to consume more sugars, sweet foods (Duffy, 2007; Turner-McGrievy, Tate, Moore, \& Popkin, 2013) and alcohol (Kampov-Polevoy, Garbutt, \& Janowsky, 1997; Kampov-Polevoy, Garbutt, \& Khalitov, 2003; Kampov-Polevoy, Tsoi, Zvartau, Neznanov, \& Khalitov, 2001; Kranzler, Sandstrom, \& Van Kirk, 2001). Thus, sweet preference may increase vulnerability to overeating or binge eating foods that are commonly reported in binge-eating episodes (Yanovski et al., 1992).

Specific taste preferences and perceptions also appear to be associated with weight status and may thus have implications for the association between sweet liking and BED. For example, Arlt, Smutzer, and Chen (2017) found that overweight individuals with BED perceived three different types of taste (quinine hydrochloride, sucrose, and 6-npropylthiouracil) as less intense than normal weighted individuals with BED and overweight healthy controls. A higher propensity for sweet and creamy tastes has also been associated with longitudinal weight gain among Pima Indians (an obesity-prone population; Salbe, DelParigi, Pratley, Drewnowski, \& Tataranni, 2004). Furthermore, women in a non-clinical sample who gained weight over six months tended to have hypo-functioning reward neural circuitry after consuming palatable foods than those who did not gain weight (Stice, Yokum, Blum, \& Bohon, 2010). Thus, it is important to examine weight status when investigating the complexities of sweet taste in individuals with BED.

Although sweet taste preference may place an individual at greater risk for binge eating, several key questions remain regarding the association between sweet taste preference's association with BED symptoms, such as binge-eating frequency, food cravings, metabolic functions, and macronutrient composition of foods consumed. The present study is preliminary and provides an investigates of sweet taste preference in BED to add to the limited extant literature (Arlt et al., 2017); thus, the statistical aims are to (1) estimate effect size and variability of sweet preference status on outcome variables and (2) to test proof-ofconcept to justify fuller pursuit of sweet taste preference in BED. Specifically, this study aims to compare the highest sweet preferers (HSP; those who prefer the highest concentration of sucrose) and other sweet preferers (OSP; those who prefer all other levels of sucrose concentration) on binge-eating frequency, over-eating episodes, BMI, food cravings, nutrient intake, and insulin-glucose regulation in a sample of participants with BED. Although we did not predict significant differences with our small sample size, we expect to see results in predicted directions. We expect that participants would evidence observably higher levels of sweet taste preference and that binge-eating frequency would show positive associations with sweet taste preference. We also proposed that HSP would show evidence of more binge-eating episodes than OSP. To clarify associations among sweet taste preference and binge-eating, we also analyzed associations between over-eating episodes that do not meet criteria for binge-eating and sweet taste. Again, we expected that HSP would show evidence of food cravings, no matter what type, more than OSP. Given our secondary aim to explore potential nutrient intake differences depending on sweet preference status, we also explored whether HSP would consume more calories overall as well as more calories from fat and carbohydrates than OSP. We also predicted that HSP show evidence of greater postprandial change in insulin and glucose levels (after ingesting a glucose solution) than OSP. A third aim of the study was to examine if HSP and OSP differ on diabetic status based on responses to an oral glucose tolerance test.

\section{Method}

Participants in this study comprised individuals from the Binge Eating and Chromium (BEACh) study (see Brownley, Von Holle, Hamer, La Via, \& Bulik, 2013). Of the 220 individuals who were self-referred and pre-screened for the BEACh study, 41 completed formal screening, which included sweet taste testing, a structured clinical interview for $\mathrm{BED}$, and evaluation of inclusion and exclusion criteria. Inclusion criteria were a Diagnostic and Statistical Manual Fourth Edition (DSM-IV; APA, 2000) BED criteria determined using the Structured Clinical Interview for DSM-IV (SCID-I/P, with Psychotic Screen; First, Spitzer, Gibbon, \& Williams, 2010) and a BMI between 25 and $45 \mathrm{~kg} / \mathrm{m}^{2}$. Exclusion criteria included: use of any medication that controls/influences glucose metabolism, insulin, appetite, or weight; fasting glucose level $>126 \mathrm{mg} / \mathrm{dL}$; use of psychotropic medication except for stable monotherapy on citalopram, escitalopram, fluoxetine, fluvoxamine, paroxetine, or sertraline. Of these 41 individuals who completed formal screening, 31 met criteria for BED but 3 declined to participate and 2 did not complete further testing to establish baseline measures, leaving 26 study participants. The Institutional Review Board at the University of North Carolina at Chapel Hill (UNC-CH) approved this study.

\subsection{Study design}

This study represents a secondary investigation of the parent BEACh study (Brownley et al., 2013), which is described in detail elsewhere. The 26 individuals with BED all completed sweet taste testing following an overnight fast in the Clinical and Translational Research Center at UNC-CH. Approximately 1-2.5 months later, subjects completed baseline testing including a 24-hour dietary recall $(N=26)$, food craving questionnaire $(n=25)$ and an oral-glucose tolerance test $(n=21)$.

\subsection{Sweet taste test}

To assess sweet taste liking, each participant, after an overnight fast and prior to the oral glucose tolerance test, tasted five concentrations of sucrose solution $(0.05,0.10,0.21,0.42$, and $0.83 \mathrm{M})$ five times in a pseudorandom order (25 total tastings). For comparison, Coca-Cola ${ }^{\circledR}$ is a $0.33 \mathrm{M}$ solution. After tasting then spitting out each solution and rinsing, the participant rated its intensity and pleasantness on a 200 $\mathrm{mm}$ analogue scale by responding to two questions: (1) "How sweet was the taste?" (intensity = "Not sweet at all" to "Extremely sweet"), and (2) "How much do you like the taste?" (pleasantness = "Disliked very much" to "Liked very much"). Average scores for each solution were used to calculate a sweet taste slope score (i.e., standardized beta). Higher slopes indicate greater pleasantness ratings as sweet concentrations increased. To determine the preferred concentration, we averaged the five pleasantness scores for each tested solution; the solution with the highest average score was considered a preferred solution. Based on previously established criteria (Damiano et al., 2014; Kampov-Polevoy et al., 2003), HSP were defined as giving the highest pleasantness rating to the highest sucrose concentration $(0.83 \mathrm{M}$ : $N=18)$; all others were defined as OSP $(N=23)$.

\subsection{Oral glucose tolerance test}

Each participant consumed a glucose solution standardized for body weight at $1.75 \mathrm{~g} / \mathrm{kg}$ (Clinical and Translational Science Institute, 2012). Blood samples were obtained via an intravenous catheter at minute 0 (fasted) and then minutes 30, 60, 90, and 120 after ingestion of the glucose solution. At McLendon Clinical Laboratories (UNC Hospitals), plasma glucose was assayed using a Vitros 5,1 FS Chemistry System (Ortho Clinical Diagnostics). Insulin was measured using a competitive radioimmunoassay (Diagnostic Systems Labs, Webster, TX) at the UNC Endocrine Lab. Assay sensitivity was $1.3 \mathrm{uIU} / \mathrm{mL}$ with a standard range of $5-300 \mu \mathrm{IU} / \mathrm{mL}$.

\subsection{4-Hour dietary recall}

A trained interviewer from the UNC-CH Nutrition Obesity Research Center Diet and Physical Activity Core contacted each participant by phone on three occasions within a maximum 2-week period to conduct 
3 separate 24-hour dietary recalls covering 2 typical weekdays and one weekend day. Use of a standardized script and multi-pass approach was employed to ensure consistency and standardization during the diet interview. Briefly, all foods, beverages, preparation methods, amounts, and recipes reported by the subject were entered to obtain an estimate of nutrient intake. To reflect the marketplace throughout the study, dietary intake data were collected and analyzed using Nutrition Data System for Research software version 2008 and 2009 developed by the Nutrition Coordinating Center, University of Minnesota, Minneapolis, MN. Final calculations were completed using the Nutrition Data System for Research (NDSR) version 2009 (3/2011). The NDSR time-related database updates analytic data while maintaining nutrient profiles true to the version used for data collection. All data passed through a 3-layer quality assurance cleaning process that included an immediate postinterview review of the entire recall to resolve any errors or unknowns, subsequent re-check of $10 \%$ of the entire dataset upon completion of data collection and, finally, scanning software outputs to identify outliers.

\subsection{Measures}

Eating Disorder Examination-Questionnaire (EDE-Q; Fairburn \& Beglin, 1994) was used to assess the frequency of disordered eating behaviors. An overall global score is obtained and was used as a proxy for eating disorder symptomatology severity in the past 28 days (higher scores indicate greater severity). The global score has high internal consistency and test-retest reliability (Luce \& Crowther, 1999). Questions number 17 (Have there been times when you have eaten what other people would regard as an unusually large amount of food?), number 18 (How many such episodes have you had over the past 28 days?), and number 19 (During how many of these episodes of overeating did you have a sense of having lost control?) of the EDE-Q were used to assess binge-eating episodes; the responses for number 19 were used as the number of binge-eating episodes over the prior 28 days given the DSM-IV criteria for binge eating (APA, 2000), and over-eating episodes were measured with number 18.

Food Craving Inventory (FCI; White, Whisenhunt, Williamson, Greenway, \& Netemeyer, 2002) is a 28 -item, self-report inventory regarding frequency of cravings for specific food items. The FCI includes subscales for carbohydrate cravings, sweet cravings, high fat cravings, fast food cravings, and a total cravings score. The FCI subscales have acceptable reliability and test-retest reliability. The psychometric properties have been confirmed for individuals with BED (White et al., 2002; White \& Grilo, 2005).

Covariates. Age (Bartoshuk, Rifkin, Marks, \& Bars, 1986; Salbe et al., 2004), sex (Kampov-Polevoy et al., 2006; Laeng, Berridge, \& Butter, 1993), BMI (Arlt et al., 2017; weight and height measured with a digital scale with stadiometer), and duration of illness (Oberndorfer et al., 2013) have been found to be associated with sweet taste preferences and were screened for inclusion as covariates. Given that previous research suggests that taste preferences differ by and are associated with sex as cited above, sensitivity analyses were run solely with female participants. The results did not differ in significance and effect sizes only varied slightly on two variables compared with analyses ran with the entire sample. Due to these negligible changes in results and the fact that removing men from the analyses decreased power, analyses that include the entire sample are presented.

\subsection{Data analysis}

Repeated measures analysis of variance (ANOVA) were used to compare pleasantness, intensity, and sweet rating within participants over the various sucrose solutions. When investigated dichotomously, linear regressions were performed to compare individuals identified as HSP vs. OSP on and binge-eating frequency, over-eating episodes, food cravings, nutrient intake, glucose and insulin regulation, and EDE-Q global score. When investigated continuously, linear regression was used to analyze the association between sweet taste slope score and these same outcome variables. Significance testing was set at an alpha of 0.05. Due to the preliminary nature of this study and the small sample size, we report and interpret partial eta squared $\left(\eta p^{2}\right)$ effect sizes using Cohen's (Cohen, 1988) suggested interpretations of small (0.01), medium (0.06), and large (0.14). Thus, while both $p$-values and effect sizes are reported, the primary metric to interpret are the effect sizes because the significance tests are underpowered in this pilot study. All statistical analyses were done with SAS/STAT software, version 9.4 (SAS Institute, 2013).

Glucose and insulin area under the curve (AUC) were calculated using the trapezoidal rule. The insulin sensitivity index (ISI) was evaluated using the Matsuda Index (Matsuda \& DeFronzo, 1999) by the formula: $10,000 / \sqrt{ }$ (fasting glucose $\times$ fasting insulin $\times$ mean glucose $\times$ mean insulin) where 10,000 simply represents a constant that allows one to obtain numbers ranging from 0 to 12 . Square root conversion was used to correct the non-linear distribution of values (Matsuda \& DeFronzo, 1999).

Individuals were classified as nondiabetic, prediabetic, or diabetic based on their fasting and 2-hour postprandial glucose values using previously established guidelines (Table 1; Phillips, 2012; World Health Organization [WHO], 2016). Prior to analysis, glucose values were transformed from $\mathrm{mg} / \mathrm{dL}$ to $\mathrm{Mmol} / \mathrm{L}$ using the formula: $\mathrm{mg} / \mathrm{dL}$ value $\times 0.0555$. Group differences in diabetes status was determined by Fisher's exact test.

\section{Results}

\subsection{Characteristics of the sample}

Eighty-five percent of the sample $(N=22)$ were female and most were White $(88 \%, 8 \%$ African American, 4\% other). The $46 \%$ that preferred the sweetest solution were classified as HSP $(N=12)$ and the rest were classified as OSP $(N=14)$. Table 2 contains additional information regarding sample characteristics; average BMI was in the obese range and past 28-day binge-eating frequency in the current sample is consistent with previous studies of BED (Reas, Grilo, \& Masheb, 2006) and non-clinical samples (Mond, Hay, Rodgers, \& Owen, 2006).

\subsection{Sweet taste preference in $B E D$}

Participants generally rated the higher sucrose concentration solutions as more pleasant $(F(4,100)=18.68, p<0.001)$ and more intense $(F(4,100)=263.74, p<0.001)$. The majority of individuals rated the first sweetest $(48 \%, N=12)$ or second sweetest $(40 \%$, $N=10$ ) solution as their preferred sweet level. The mean slope score was $0.55(S D=0.61)$.

\subsection{Covariates}

Age, sex, BMI, and duration of illness were screened for inclusion by including them in the models that follow and retaining those in the final models that demonstrated an association with the dependent variable of partial eta squared $>0.01$ [small]. Duration of illness could not be considered as a covariate with the dependent variables binge-eating

Table 1

Interpretation of oral glucose tolerance test (Mmol/L).

\begin{tabular}{llll}
\hline & Fasting & $2 \mathrm{~h}$ & Implications \\
\hline No diabetes & $\leq 6.0$ & $<7.8$ & No excess micro- nor macro-vascular risk \\
Prediabetes & $6.1-6.9$ & $7.8-11.0$ & Excess macro- but not micro-vascular risk \\
Diabetes & $\geq 7.0$ & $\geq 11.1$ & Excess macro- and micro-vascular risk \\
\hline
\end{tabular}




\begin{tabular}{|c|c|c|c|c|c|c|c|c|c|c|}
\hline & \multicolumn{4}{|c|}{$\begin{array}{l}\text { HSP } \\
(n=12)\end{array}$} & \multicolumn{4}{|c|}{$\begin{array}{l}\text { OSP } \\
(n=14)\end{array}$} & \multicolumn{2}{|l|}{ Total } \\
\hline & $M$ & $S D$ & Min & Max & $M$ & $S D$ & Min & Max & $M$ & $S D$ \\
\hline Age & 33.33 & 10.29 & 18 & 51 & 42.07 & 11.19 & 20 & 57 & 38.04 & 11.46 \\
\hline BMI & 34.77 & 5.54 & 24.09 & 42.57 & 34.25 & 5.75 & 26.19 & 43.56 & 34.49 & 5.55 \\
\hline EDE-Q global score & 3.32 & 1.04 & 1.89 & 5.35 & 3.38 & 0.95 & 0.81 & 4.54 & 3.35 & 0.97 \\
\hline Binge-eating frequency & 25.08 & 21.97 & 8 & 80 & 13.07 & 6.15 & 0 & 21 & 18.62 & 16.41 \\
\hline
\end{tabular}

BMI = Body mass index.

EDE-Q = Eating Disorder Examination - Questionnaire.

HSP $=$ highest sweet preferers; OSP $=$ other sweet preferers.

frequency, over-eating frequency, and global eating disorder symptoms due to the timing of the assessments. These dependent variables were collected at screening whereas duration of illness was collected at a later baseline assessment.

\subsection{Sweet taste preference and binge-eating}

Zero-order correlations between binge-eating frequency and hedonic ratings for each solution ranged from 0.37 ( $0.83 \mathrm{M}$ solution) to $-0.20(0.05 \mathrm{M}$ solution) and were not statistically significant ( $p s>0.05$ ). Compared with OSP, HSP reported statistically higher binge-eating frequency $(25.1 \pm 22.0$ vs. $13.1 \pm 6.15$ episodes; $F(1$, $\left.23)=6.52, \quad p=0.02, \quad \eta p^{2}=0.22\right) \quad$ and over-eating frequency $(26.7 \pm 21.2$ vs. $13.6 \pm 5.98$ episodes; $F(1,23)=7.24, p=0.01$, $\left.\eta p^{2}=0.24\right)$ in the past 28 days. There was a small effect of binge-eating frequency $\left(\beta=0.21 ; \eta p^{2}=0.04, p=0.37\right)$ and over-eating frequency $\left(\beta=0.23 ; \eta p^{2}=0.04 ; p=0.33\right)$ being positively associated with sweet taste preference ratings. The associations were statistically nonsignificant.

\subsection{Sweet taste preference and food cravings}

The effect sizes for group differences in FCI scores were uniformly small ( $\eta p^{2} \leq 0.001$ to 0.05 ; see Table 3 ) and mean differences were not statistically significant. Specifically, the HSP and OSP groups did not differ on total cravings $(F(1,20)=0.02, p=0.88)$, fast-food fat craving $(F(1,19)=0.97, p=0.34)$, carbohydrate craving $(F(1,19)=0.04$, $p=0.85)$, sweet food craving $(F(1,21)=0.12, p=0.74)$, or high fat food craving $(F(1,19)=0.01, p=0.99)$.

When measured continuously, total cravings $(\beta=0.04$; $\left.\eta p^{2}=0.002 ; p=0.83\right), \quad$ cravings for fast-food fats $(\beta=0.21$; $\left.\eta p^{2}=0.06 ; p=0.30\right)$, and cravings for food high in fats $(\beta=0.24$; $\eta p^{2}=0.08 ; p=0.22$ ) were positively associated with sweet taste with negligible-medium effect sizes. Cravings for carbohydrates

Table 3

Mean $(M)$ and standard deviation $(S D)$ for Food Craving Inventory total and scale scores and effect sizes in high sweet preferers (HSP) and other sweet preferers (OSP) with bingeeating disorder.a

\begin{tabular}{|c|c|c|c|c|c|}
\hline & \multicolumn{2}{|c|}{$\begin{array}{l}\text { HSP } \\
(n=11)\end{array}$} & \multicolumn{2}{|c|}{$\begin{array}{l}\text { OSP } \\
(n=14)\end{array}$} & \multirow{2}{*}{$\begin{array}{l}\text { Effect size } \\
\eta p^{2}\end{array}$} \\
\hline & $M$ & $S D$ & $M$ & $S D$ & \\
\hline Total craving score & 69.27 & 16.63 & 70.63 & 16.65 & 0.001 \\
\hline Fast-food fats & 12.73 & 2.90 & 11.71 & 3.79 & 0.05 \\
\hline Carbohydrates/starches & 19.00 & 5.35 & 19.86 & 7.08 & 0.002 \\
\hline Sweets & 23.00 & 9.32 & 22.64 & 6.07 & 0.006 \\
\hline High fat & 14.55 & 6.22 & 16.29 & 6.63 & $<0.001$ \\
\hline
\end{tabular}

HSP $=$ highest sweet preferers; OSP $=$ other sweet preferers.

${ }^{\text {a }}$ Interpretation of partial eta squared based on Cohen (1988): small (0.01), medium (0.06), and large (0.14) $\left(\beta=-0.17 ; \quad \eta p^{2}=0.05 ; \quad p=0.36\right) \quad$ and sweets $(\beta=-0.02$; $\left.\eta p^{2} \leq 0.001 ; p=0.93\right)$ were negatively associated with sweet taste with negligible-small effect sizes. No associations were statistically significant.

\subsection{Sweet taste preference, BMI, eating disorder symptomatology, and} nutrient intake

Mean BMI did not differ significantly between HSP $(M=34.4 \pm 5.38)$ and OSP $(M=34.6 \pm 6.28 ; F(1,39)=0.01$, $\left.p=0.92, \eta p^{2}=0.0003\right)$ even when adjusting for sex and age $(F(1,38)$ $\left.=0.01, p=0.90, \eta p^{2}=0.0004\right)$. Likewise, mean global EDE-Q did not differ between HSP $(M=3.3 \pm 1.00)$ and OSP $(M=3.4 \pm 0.95 ; F(1$, $\left.37)=0.08, p=0.78, \eta p^{2}=0.003\right)$. When measured continuously, global EDE-Q was positively associated with sweet taste with a small effect size, though the association was not statistically significant $\left(\beta=0.12 ; \eta p^{2}=0.02 ; p=0.61\right)$.

Table 4 displays nutrient intake for the HSP and OSP groups. The effect sizes were large for differences in 24-hour caloric intake, protein intake, and medium for fat intake. The mean differences in total calories and protein calories was significantly different. Specifically, compared with OSP, HSP reported consuming approximately 847 more total calories per day $\left(F(1,23)=4.37, p=0.04, \eta p^{2}=0.16\right)$, reflecting approximately 114 more protein calories $(F(1,23)=4.48, p=0.04$, $\left.\eta p^{2}=0.16\right), 335$ more fat calories $(F(1,20)=1.72, p=0.20$, $\left.\eta p^{2}=0.08\right)$, and 398 more carbohydrate calories $(F(1,23)=3.67$, $\left.p=0.07, \eta p^{2}=0.14\right)$. HSP and OSP did not differ significantly in percentage of calories from fat $(F(1,20)=0.63, p=0.44)$, carbohydrate $(F(1,21)=0.14, \quad p=0.71)$, or protein $(F(1,20)=0.04$, $p=0.84)$, or in alcohol calorie intake $(F(1,24)=0.15, p=0.70)$.

When measured continuously, all types of caloric intake were

Table 4

Mean $(M)$ and standard deviation (SD) for daily nutrient intake and effect sizes in high sweet preferers (HSP) and other sweet preferers (OSP) with binge-eating disorder.

\begin{tabular}{|c|c|c|c|c|c|}
\hline & \multicolumn{2}{|l|}{$\begin{array}{l}\text { HSP } \\
(n=12)\end{array}$} & \multicolumn{2}{|l|}{$\begin{array}{l}\text { OSP } \\
(n=14)\end{array}$} & \multirow{2}{*}{$\begin{array}{l}\begin{array}{l}\text { Effect } \\
\text { Size }^{\mathrm{a}}\end{array} \\
\eta p^{2}\end{array}$} \\
\hline & $M$ & $S D$ & $M$ & $S D$ & \\
\hline Total caloric intake (kcals) & 2972.17 & 1316.84 & 2125.06 & 386.96 & 0.16 \\
\hline Fat intake (kcals) & 1072.92 & 502.67 & 728.50 & 389.48 & 0.08 \\
\hline Carbohydrate intake (kcals) & 1441.89 & 673.63 & 1044.12 & 108.05 & 0.14 \\
\hline Protein intake (kcals) & 440.95 & 179.34 & 326.99 & 52.73 & 0.16 \\
\hline Alcohol intake (kcals) & 18.05 & 39.33 & 25.28 & 7.44 & 0.006 \\
\hline$\%$ Caloric intake from fat & 35.85 & 4.17 & 33.20 & 7.74 & 0.03 \\
\hline $\begin{array}{l}\text { \% Caloric intake from } \\
\text { carbohydrate }\end{array}$ & 48.22 & 4.92 & 49.03 & 5.32 & 0.007 \\
\hline $\begin{array}{l}\% \text { Caloric intake from } \\
\text { protein }\end{array}$ & 15.40 & 3.56 & 16.10 & 57.83 & 0.002 \\
\hline
\end{tabular}

HSP = highest sweet preferers; OSP = other sweet preferers.

a Interpretation of partial eta squared based on Cohen (1988): small (0.01), medium (0.06), and large (0.14). 
Table 5

Mean $(M)$ and standard deviation $(S D)$ for postprandial blood glucose area under the curve (AUC), insulin AUC, and insulin sensitivity index in high sweet preferers (HSP) and other sweet preferers (OSP) with binge eating disorder who completed an oral glucose tolerance test $(\mathrm{n}=21)$.

\begin{tabular}{|c|c|c|c|c|c|}
\hline & \multicolumn{2}{|l|}{ HSP } & \multicolumn{2}{|l|}{ OSP } & \multirow{2}{*}{$\frac{\text { Effect size }^{\mathrm{a}}}{\eta p^{2}}$} \\
\hline & $M$ & $S D$ & $M$ & $S D$ & \\
\hline Postprandial glucose AUC & 222.39 & 50.58 & 319.30 & 97.75 & 0.16 \\
\hline Postprandial insulin AUC & 136.61 & 70.40 & 329.62 & 240.91 & 0.27 \\
\hline Insulin sensitivity index (ISI) & 1.83 & 1.34 & 2.96 & 1.14 & 0.24 \\
\hline
\end{tabular}

HSP $=$ highest sweet preferers; OSP $=$ other sweet preferers

${ }^{a}$ Interpretation of partial eta squared based on Cohen (1988): small (0.01), medium (0.06), and large (0.14).

positively associated with sweet taste preference except percentage of caloric intake from carbohydrates; effect sizes ranged from negligiblemedium: total calories $\left(\beta=0.09 ; \eta p^{2}=0.009 ; p=0.68\right)$, fat calories $\left(\beta=0.04 ; \eta p^{2}=0.002 ; p=0.84\right)$, carbohydrate calories $(\beta=0.08$; $\left.\eta p^{2}=0.007 ; p=0.71\right)$, protein calories $\left(\beta=0.21 ; \eta p^{2}=0.06\right.$; $p=0.28)$, alcohol calories $\left(\beta=0.09 ; \eta p^{2}=0.008 ; p=0.67\right)$, percent caloric intake from fat $\left(\beta=0.05 ; \eta p^{2}=0.005, p=0.76\right)$, percent caloric intake from protein $\left(\beta=0.07 ; \eta p^{2}=0.006 ; p=0.76\right)$. Percent of caloric intake from carbohydrates was inversely related to sweet taste with a small effect size $\left(\beta=-0.14 ; \eta p^{2}=0.02 ; p=0.57\right)$. None of the associations reached statistical significance.

\subsection{Sweet taste preference, glucose, and insulin regulation}

A subset of 21 participants completed the oral glucose tolerance test. Table 5 displays mean postprandial blood glucose levels changes, insulin level changes, and insulin sensitivity indexes. HSP and OSP exhibited similar basal serum insulin and glucose levels but responded differently to the oral glucose challenge. Compared with OSP, HSP exhibited a significantly smaller insulin response insulin $_{\mathrm{AUC}}(F(1,16)$ $=5.98, p=0.02, \eta p^{2}=0.27$ ); Fig. 1] and numerically smaller glucose response [glucose AUC $\left(F(1,16)=3.08, p=0.10, \eta p^{2}=0.16\right)$; Fig. 2] and had significantly higher insulin sensitivity $(\mathrm{F}(1,16)=4.95$, $p=0.04, \eta p^{2}=0.24$ ).

When measured continuously, glucose response AUC $(\beta=-0.28$; $\left.\eta p^{2}=0.11 ; p=0.18\right)$ and insulin response AUC $(\beta=-0.40$; $\eta p^{2}=0.31 ; p=-0.40$ ) were negatively associated with sweet taste, such that individuals who preferred sweeter solutions had smaller postprandial glucose and insulin AUC responses (effect sizes ranged from medium-large). Insulin sensitivity was positively associated with sweet taste at a large effect size $\left(\beta=0.36 ; \eta p^{2}=0.18 ; p=0.08\right)$. The association with insulin response AUC $\left(\beta=-0.40 ; \eta p^{2}=0.18\right.$; $p=0.02$ ) was statistically significant, but the other associations were not.

\subsection{Sweet taste preference and diabetes status}

There was no statistically significant difference between the two groups in diabetes status; $100 \%(10 / 10)$ of HSP were categorized as no diabetes; OSP were classified as 55\% (6/11) no diabetes, $9 \%(1 / 11)$ prediabetes, and $36 \%(4 / 11)(p=0.058)$ diabetes. These analyses could not be run continuously or with covariates as there was not enough variability in the diabetes status data.

\section{Discussion}

The current study explored the role of sweet taste preference in BED. Specifically, we evaluated the association between sweet taste preference and binge-eating frequency, episodes of over-eating, illnessduration, BMI, food cravings, nutrient intake, glucose and insulin regulation, and diabetes status. Due to the preliminary nature of this study, thus, being underpowered in terms of significance testing, effect sizes and their confidence intervals were interpreted to demonstrate proofof-concept.

Consistent with our a priori hypothesis, the majority of participants in our study (about $80 \%$ ) chose one of the two sweetest solutions as the most pleasurable. Forty-four percent of participants rated the highest solution as pleasurable and, thus, were categorized as HSP according to previously used protocols (Damiano et al., 2014; Kampov-Polevoy et al., 2003). Other studies investigating sweet taste preference in adults have found a slightly higher percentage of sweet likers (50\%) with no differences by sex (Kampov-Polevoy et al., 2006) or by an index of problematic drinking (Kampov-Polevoy et al., 2014). Whether or not sweet preference is, in fact, less common among individuals with BED than in the general population is unclear.

Although only one previous study has assessed taste preference in BED (Arlt et al., 2017), two previous studies examined whether sweet taste preference differentiates individuals with bulimia nervosa from healthy controls. In one small $(N=24)$ study using aspartame-sweetened solutions (Klein, Schebendach, Brown, Smith, \& Walsh, 2009) and a second small $(N=26)$ study using sucrose sweetened solutions (Drewnowski et al., 1992), individuals with bulimia nervosa indicated greater liking of the two highest sweet solutions compared with healthy controls, but these group differences were statistically significant in only one of the studies (Drewnowski, Krahn, Demitrack, Nairn, \& Gosnell, 1992). Larger-scale studies are needed to determine whether a propensity to prefer highly concentrated sweets differentiates

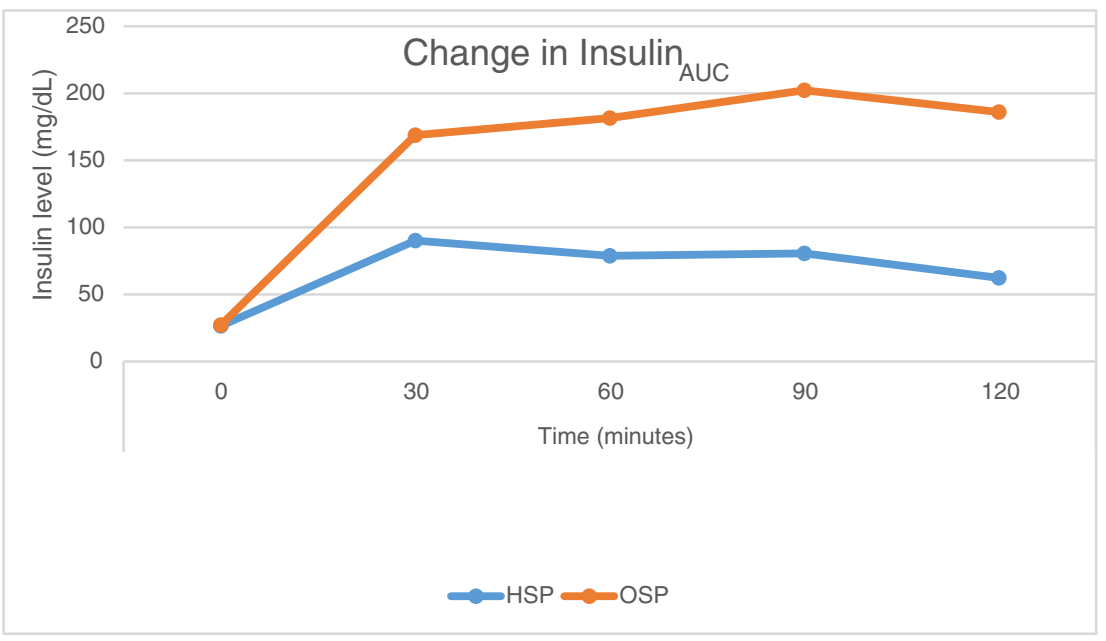

Fig. 1. Change in insulin during the oral glucose tolerance test. Note. Each participant consumed a glucose solution standardized for body weight at $1.75 \mathrm{~g} / \mathrm{kg}$ (Clinical and Translational Science Institute, 2012). Insulin was measured at minute 0 (fasted) and then minutes $30,60,90$, and 120 after ingestion of the glucose solution. HSP $=$ high sweet preferers; OSP $=$ other sweet preferers. 


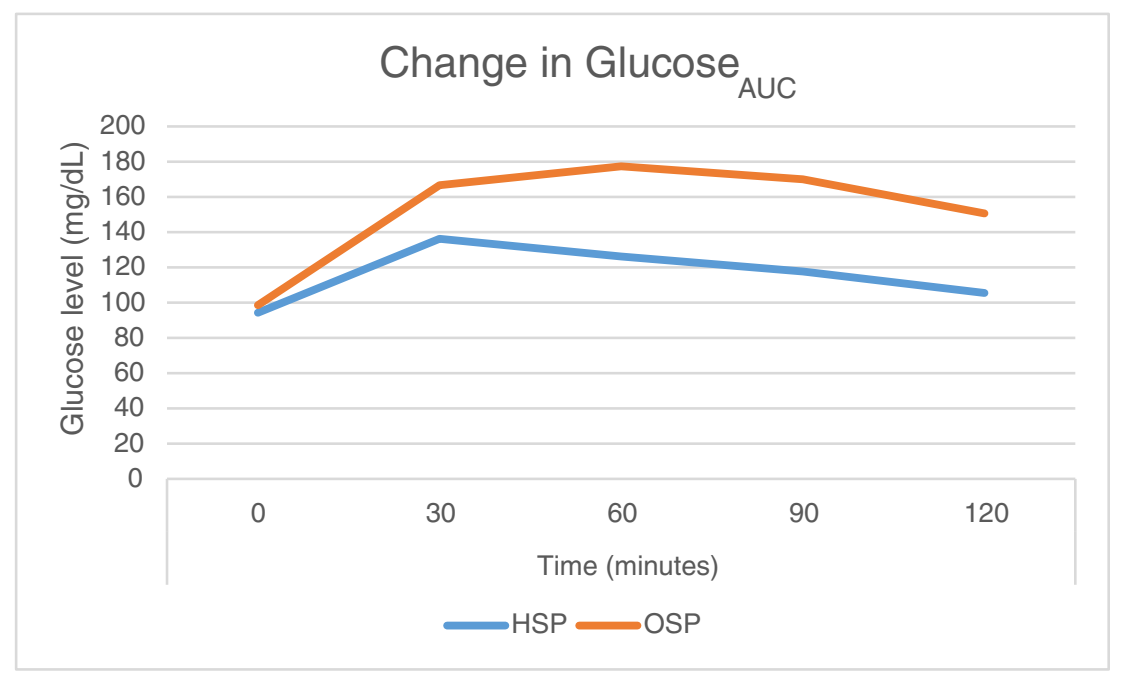

Fig. 2. Change in glucose during the oral glucose tolerance test Note. Each participant consumed a glucose solution standardized for body weight at $1.75 \mathrm{~g} / \mathrm{kg}$ (Clinical and Translational Science Institute, 2012). Glucose was measured at minute 0 (fasted) and then minutes $30,60,90$, and 120 after ingestion of the glucose solution.

HSP $=$ high sweet preferers; OSP $=$ other sweet preferers.

individuals who binge eat from those who do not and, if so, whether that propensity is clinically meaningful in patients with eating disorders.

In the current study, individuals with HSP reported approximately 6 more binge-eating episodes/month than OSP and those who reported higher sweet liking scores tended to experience more eating disorder symptomatology, binge-eating episodes, and over-eating episodes in the past 28-days. These findings indicate that sweet preference is related to both binge-eating episodes and over-eating tendencies and is consistent with previous research suggesting that sweet taste wanting and liking are associated with higher binge-eating scores (Dalton \& Finlayson, 2014; Finlayson et al., 2011). While the neurobiological underpinnings of a HSP phenotype is not well understood, there is evidence to suggest that HSP may experience opioid dysfunction with accompanying motivation to seek opioid stimulation (Calcagnetti \& Reid, 1983; Leventhal, Kirkham, Cole, \& Bodnar, 1995). Further, modulation of the opioid system via opioid antagonists (i.e., Naloxone) reduces bingeeating of palatable food (Adam \& Epel, 2007). Thus, opioid dysfunction may underlie the association between HSP and binge-eat/over-eating episodes. Future research should aim to directly measure this opioid hypothesis in disordered eating and control samples. Although preliminary, more frequent binge-eating episodes in HSP observed in the current study may be due to underlying psychological motives, such as emotional eating. Because higher binge-eating frequency has been associated with more severe EDs and comorbid psychopathology (i.e., depression) in individuals with BED (Grilo, Ivezaj, \& White, 2015), future studies should investigate whether sweet taste preference is a harbinger for higher binge-eating frequency and ED symptom severity in BED.

As hypothesized, OSP reported consuming fewer calories $(\sim 2100 \mathrm{kcal} /$ day $)$ compared with HSP $(\sim 3000 \mathrm{kcal} /$ day $)$. Continuous analyses yielded that preference for sweeter solutions was associated with increased protein intake and decreased carbohydrate intake; all other associations were negligible. The macronutrient intake for both HSP and OSP groups was approximately $50 \%$ carbohydrate, $35 \%$ fat, and $15 \%$ protein; and alcohol intake was generally quite low $(\sim 1 \%)$. The caloric intake of the HSP group is notable because it exceeds the recommended daily caloric intake for an adult woman (1600-2400 kcal/day; U.S. Department of Agriculture \& U.S. Department of Health and Human Services, 2010) and suggests that preference for highly concentrated sweets may play a role in weightrelated outcomes in BED. The low alcohol intake observed in the current study is notable in light of data suggesting that alcohol use is quite common (65.2\%) in BED (Fouladi et al., 2015). Future investigations of sweet preference, alcohol use, and weight in BED could benefit from including measures of impulsivity, which is a well-established feature of binge-spectrum EDs and alcohol use disorders (Crews \& Boettiger, 2009; Schag, Schonleber, Teufel, Zipfel, \& Giel, 2013). Finally, although both HSP and OSP endorsed cravings for sweets to a greater extent than for fats or carbohydrates, we found no discernible differences in food cravings within or between groups, which appears consistent with previous results suggesting no clear pattern of specific food cravings in BED (Yanovski, 2003).

The current study explored a previously under-investigated area of postprandial insulin-glucose regulation in individuals with BED. HSP and OSP had similar mean fasting insulin and glucose levels; but, HSP exhibited smaller postprandial insulin and glucose responses than OSP and were slightly more insulin sensitive and, thus, fewer HSP were classified as prediabetic/diabetic $(N=0 / 10)$ than OSP $(N=5 / 11)$. When measured continuously, similar results emerged: those who reported higher sweet taste preferences tended to have increased insulin sensitivity and smaller changes in postprandial glucose and insulin responses. This is contrary to the literature suggesting an increase in postprandial glucose and insulin levels in the presence of diets high in sucrose foods (O'Keefe \& Bell, 2007). Together, these findings paint an uncertain picture wherein sweet liking may be differentially associated with the behavioral and the physiological manifestations of BED.

This study had several limitations in addition to being underpowered for significance testing and lacking a non-BED control group. The categorization of HSP and OSP, while based on some previous sweet taste preference methodology (Damiano et al., 2014; KampovPolevoy et al., 2003), is not characteristic of all sweet taste preference studies. For example, some studies include highest preference ratings for the top two concentrations of sucrose solutions ( $0.42 \mathrm{M}$ and $0.83 \mathrm{M}$ ) in the "sweet likers" category (Kampov-Polevoy et al., 1997; Looy, Callaghan, \& Weingarten, 1992). Just as well, previous research has shown that the sucrose recognition and intensity thresholds among a large sample of non-ED individuals ranges from $0.01 \mathrm{M}$ to $1.8 \mathrm{M}$ (Bartoshuk et al., 1986), thus potentially making our 0.8 cut-off seem somewhat arbitrary, especially since $90 \%$ of our sample preferred solutions that were sweeter than Coca-Cola. Future studies should take such sucrose thresholds into account when devising and administering sweet taste tests. We did not utilize a standardized gustation measure, such as the Gustation Assessment in the NIH Toolbox (see Coldwell et al., 2013), which prevented us from screening normative gustation differences at the population level; it would behoove future studies to utilize such an assessment in order to rule out known normalized differences. Another methodological factor that may have implications regarding our findings is that the sweet taste test occurred after participants completed an overnight fast; that is, they were not satiated 
when completing the test. Previous research suggests that hunger and satiety may be associated with taste perceptions. Given that hunger and satiety was not measured before/after the sweet taste test (Laeng et al., 1993), we are unable to rule out this as a confounding factor. Thus, future studies should investigate the relationship between fasting and satiety states and their associations with sweet taste preference in order to clarify these complex relationships.

As for all results of this secondary-analysis study, our findings are preliminary and must be interpreted with great caution. At best, they provide impetus for further investigation of sweet taste preference in BED; that is, many of the analyses revealed medium-large effect sizes, which provides a basis for future investigation into the associations tested in this study. As binge eating is the hallmark feature of BED, mechanisms that amplify binge eating need insight. Our data may be useful for estimating the appropriate sample size for future studies, taking into consideration the analytical challenges of differential loss of invasively-derived physiological measures (e.g., blood insulin and glucose) and self-report or interview-based behavioral data. Future research may consider including individuals with bulimia nervosa as well as healthy and psychiatric controls in order to better understand the unique and additive effects of sweet taste preference on binge-eating behavior and its related metabolic health outcomes.

\section{Conclusions}

The results from our preliminary study suggest an association between individuals with BED who also have a preference for foods high in sucrose and a greater likelihood of binge eating more frequently. Thus, these particular individuals may also experience excess nutrient intake independent of underlying glucose regulation status. It remains unknown whether sweet taste preference is associated with or affects weight or metabolic health in individuals with BED over time.

\section{Conflict of interest and source of funding}

Dr. Bulik is a grant recipient from and consultant for Shire Pharmaceuticals and acknowledges funding from the Swedish Research Council (VR Dnr: 538-2013-8864); Dr. Baker was supported by grant NIH K01MH106675; Dr. Peat is a consultant for Sunovion Pharmaceuticals

\section{Acknowledgments}

This study received support in the form of trained interviewers from the Diet, Physical Activity and Body Composition Core in the UNC Nutrition Obesity Research Center; grant DK56350. Study visits were conducted in the North Carolina Translational \& Clinical Sciences Institute (UL1TR001111). Dr. Bulik acknowledges funding from the Swedish Research Council (VR Dnr: 538-2013-8864).

\section{References}

Adam, T. C., \& Epel, E. S. (2007). Stress, eating and the reward system. Physiology \& Behavior, 91, 449-458. http://dx.doi.org/10.1016/j.physbeh.2007.04.011.

American Psychiatric Association (2000). Diagnostic and statistical manual of mental disorders (4th ed.). Washington, DC: Author Text rev.

American Psychiatric Association (2013). Diagnostic and statistical manual of mental disorders (5th ed). Washington, DC: Author.

Arlt, J. M., Smutzer, G. S., \& Chen, E. Y. (2017). Taste assessment in normal weight and overweight individuals with co-occurring binge eating disorder. Appetite, 113, 239-245. http://dx.doi.org/10.1016/j.appet.2017.02.034.

Avena, N. M., Rada, P., \& Hoebel, B. G. (2009). Sugar and fat bingeing have notable differences in addictive-like behavior. Journal of Nutrition, 139, 623-628. http://dx. doi.org/10.3945/jn.108.097584.

Bartoshuk, L. M., Rifkin, B., Marks, L. E., \& Bars, P. (1986). Taste and aging. Journal of Gerontology, 41, 51-57.

Brownley, K. A., Von Holle, A., Hamer, R. M., La Via, M., \& Bulik, C. M. (2013). A doubleblind, randomized pilot trial of chromium picolinate for binge eating disorder: Results of the Binge Eating and Chromium (BEACh) Study. Journal of Psychosomatic Research, 75, 36-42. http://dx.doi.org/10.1016/j.jpsychores.2013.03.092.
Calcagnetti, D. J., \& Reid, L. D. (1983). Morphine and acceptability of putative reinforcers. Pharmacology, Biochemistry, and Behavior, 18, 567-569.

Clinical and Translational Science Institute. (2012). Standard operating procedures. Retrieved from https://ctsi.ucla.edu/researcher-resources/files/docs/sop-ogtt.pdf.

Cohen, J. (1988). Statistical power analysis for the behavioral sciences (2nd ed.). Hillsdale, NJ: Lawrence Earlbaum Associates.

Coldwell, S. E., Mennella, J. A., Duffy, V. B., Pelchat, M. L., Griffith, J. W., Smutzer, G., Cowart, B. J., ... Hoffman, H. J. (2013). Gustation assessment using the NIH Toolbox. Neurology, 30, S20-S24. http://dx.doi.org/10.1212/wnl.0b013e3182872e38.

Crews, F. T., \& Boettiger, C. A. (2009). Impulsivity, frontal lobes and risk for addiction. Pharmacology Biochemistry and Behavior, 93, 237-247. http://dx.doi.org/10.1016/j. pbb.2009.04.018.

Dalton, M., \& Finlayson, G. (2014). Psychobiological examination of liking and wanting for fat and sweet taste in trait binge eating females. Physiology \& Behavior, 136, 128-134. http://dx.doi.org/10.1016/j.physbeh.2014.03.019.

Damiano, C. R., Aloi, J., Burrus, C., Garbutt, J. C., Kampov-Polevoy, A. B., \& Dichter, G. S (2014). Intact hedonic responses to sweet tastes in autism spectrum disorder. Research in Autism Spectrum Disorders. 8. Research in Autism Spectrum Disorders (pp. 230-236). . http://dx.doi.org/10.1016/j.rasd.2013.12.003.

Drewnowski, A., Krahn, D. D., Demitrack, M. A., Nairn, K., \& Gosnell, B. A. (1992). Taste responses and preferences for sweet high-fat foods: Evidence for opioid involvement. Physiology \& Behavior, 51, 371-379. http://dx.doi.org/10.1016/0031-9384(92) 90155-U.

Duffy, V. B. (2007). Variation in oral sensation: Implications for diet and health. Current Opinion in Gastroenterology, 23, 171-177. http://dx.doi.org/10.1097/MOG. ob013e3280147d50.

Fairburn, C. G., \& Beglin, S. J. (1994). Assessment of eating disorders: Interview or selfreport questionnaire? The International Journal of Eating Disorders, 16(4), 363-370. http://dx.doi.org/10.1002/1098-108X(199412)16:4<363::AIDEAT2260160405 > 3.0.CO:2-\#.

Finlayson, G., Arlotti, A., Dalton, M., King, N., \& Blundell, J. E. (2011). Implicit wanting and explicit liking are markers for trait binge eating. A susceptible phenotype for over-eating. Appetite, 57, 722-728. http://dx.doi.org/10.1016/j.appet.2011.08.012.

First, M. B., Spitzer, R. L., Gibbon, M., \& Williams, J. B. (2010). Structured clinical interview for DSM-IV-TR Axis I disorders (Patient Edition). New York: New York State Psychiatric Institute.

Fouladi, F., Mitchell, J. E., Crosby, R. D., Engel, S. G., Crow, S., Hill, L., ... Steffen, K. J. (2015). Prevalence of alcohol and other substance use in patients with eating disorders. European Eating Disorders Review, 23, 531-536. http://dx.doi.org/10.1002/ erv. 2410 .

Greeno, C. G., Wing, R. R., \& Shiffman, S. (2000). Binge antecedents in obese women with and without binge eating disorder. Journal of Consulting and Clinical Psychology, 68, 95-102. http://dx.doi.org/10.1037//0022-006X.68.1.95.

Grilo, C. M., Ivezaj, V., \& White, M. A. (2015). Evaluation of the DSM-5 severity indicator for binge eating disorder in a clinical sample. Behaviour Research and Therapy, 17, 110-114. http://dx.doi.org/10.1016/j.brat.2015.05.003.

Hudson, J. I., Lalonde, J. K., Coit, C. E., Tsuang, M. T., McElroy, S. L., Crow, S. J., ... Pope, H. G. (2010). Longitudinal study of the diagnosis of components of the metabolic syndrome in individuals with binge-eating disorder. American Journal of Clinical Nutrition, 91, 1568-1573. http://dx.doi.org/10.3945/ajcn.2010.29203.

Kampov-Polevoy, A. B., Alterman, A., Khalitov, E., \& Garbutt, J. C. (2006). Sweet preference predicts mood altering effect of and impaired control over eating sweet foods. Eating Behaviors, 7, 181-187. http://dx.doi.org/10.1016/j.eatbeh.2005.09.005.

Kampov-Polevoy, A., Garbutt, J. C., \& Janowsky, D. (1997). Evidence of preference for a high-concentration sucrose solution in alcoholic men. American Journal of Psychiatry, 154, 269-270. http://dx.doi.org/10.1176/ajp.154.2.269.

Kampov-Polevoy, A. B., Garbutt, J. C., \& Khalitov, E. (2003). Family history of alcoholism and response to sweets. Alcoholism: Clinical and Experimental Research, 27, 1743-1749. http://dx.doi.org/10.1097/01.ALC.0000093739.05809.DD.

Kampov-Polevoy, A., Lange, L., Bobashev, G., Eggleston, B., Root, T., \& Garbutt, J. C. (2014). Sweet-liking is associated with transformation of heavy drinking into alcoholrelated problems in young adults with high novelty seeking. Alcoholism: Clinical and Experimental Research, 38, 2119-2126. http://dx.doi.org/10.1111/acer.12458.

Kampov-Polevoy, A. B., Tsoi, M. V., Zvartau, E. E., Neznanov, N. G., \& Khalitov, E. (2001) Sweet liking and family history of alcoholism in hospitalized alcoholic and non-alcoholic patients. Alcohol and Alcoholism, 36, 165-170. http://dx.doi.org/10.1093/ alcalc/36.2.165.

Klein, D. A., Schebendach, J. E., Brown, A. J., Smith, G. P., \& Walsh, B. T. (2009). Modified sham feeding of sweet solutions in women with and without bulimia nervosa. Physiology \& Behavior, 96, 44-50. http://dx.doi.org/10.1016/j.physbeh.2008. 08.008.

Kranzler, H. R., Sandstrom, K. A., \& Van Kirk, J. (2001). Sweet taste preference as a risk factor for alcohol dependence. American Journal of Psychiatry, 158, 813-815. http:// dx.doi.org/10.1176/appi.ajp.158.5.813.

Laeng, B., Berridge, K. C., \& Butter, C. M. (1993). Pleasantness of a sweet taste during hunger and satiety: Effects of gender and "sweet tooth". Appetite, 21, 247-254.

Leibenluft, E., Fiero, P. L., Bartko, J. J., Moul, D. E., \& Rosenthal, N. E. (1993). Depressive symptoms and the self-reported use of alcohol, caffeine, and carbohydrates in normal volunteers and four groups of psychiatric outpatients. The American Journal of Psychiatry, 150, 294-301. http://dx.doi.org/10.1176/ajp.150.2.294.

Leventhal, L., Kirkham, T. C., Cole, J. L., \& Bodnar, R. J. (1995). Selective actions of central $\mu$ and $\kappa$ opioid antagonists upon sucrose intake in sham-fed rats. Brain Research, 685, 205-210.

Looy, H., Callaghan, S., \& Weingarten, H. P. (1992). Hedonic response of sucrose likers and dislikers to other gustatory stimuli. Physiology \& Behavior, 52, 219-225.

Luce, K. H., \& Crowther, J. H. (1999). The reliability of the eating disorder examination - 
Self-report Questionnaire Version (EDE-Q). International Journal of Eating Disorders, 25, 349-351. http://dx.doi.org/10.1002/(SICI)1098-108X(199904)25:3 < 349::AIDEAT15 > 3.0.CO;2-M.

Matsuda, M., \& DeFronzo, R. A. (1999). Insulin sensitivity indices obtained from oral glucose tolerance testing: Comparison with the euglycemic insulin clamp. Diabetes Care, 22, 1462-1470. http://dx.doi.org/10.2337/diacare.22.9.1462.

Mitchell, J. E., King, W. C., Pories, W., Wolfe, B., Flum, D. R., SLaniolas, K., ... Yanovski, S. (2015). Binge eating disorder and medical comorbidities in bariatric surgery candidates. International Journal of Eating Disorders, 48, 471-476. http://dx.doi.org/10. 1002/eat.22389.

Mond, J., Hay, P., Rodgers, B., \& Owen, C. (2006). Self-recognition of disordered eating among women with bulimic-type eating disorders: A community-based study. International Journal of Eating Disorders, 39, 747-753.

Oberndorfer, T. A., Frank, G. K. W., Simmons, A. N., Wagner, A., McCurdy, D., Fudge, J. L., Yang, T. T., ... Kaye, W. H. (2013). Altered insula response to sweet taste processing after recovery from anorexia and bulimia nervosa. American Journal of Psychiatry, 170, 1143-1151. http://dx.doi.org/10.1176/appi.ajp.2013.11111745.

O'Keefe, J. H., \& Bell, D. S. (2007). Postprandial hyperglycemia/hyperlipidemia (postprandial dysmetabolism) is a cardiovascular risk factor. American Journal of Cardiology, 100, 899-904.

Phillips, P. J. (2012). Oral glucose tolerance testing. Australian Family Physician, 41(6), 291-393.

Raevouri, A., Suokas, J., Haukka, J., Gissler, M., Linna, M., Grainger, M., \& Suvisaari, J. (2015). Highly increased risk of type 2 diabetes in patients with binge eating disorder and bulimia nervosa. International Journal of Eating Disorders, 48, 555-562. http://dx. doi.org/10.1002/eat.22334.

Reas, D. L., Grilo, C. M., \& Masheb, R. M. (2006). Reliability of the Eating Disorder Examination-Questionnaire in patients with binge eating disorder. Behaviour Research and Therapy, 44(1), 43-51.

Roehrig, M., Masheb, R. M., White, M. A., \& Grilo, C. M. (2008). The metabolic syndrome and behavioral correlates in obese patients with binge eating disorder. Obesity, 17, 481-486. http://dx.doi.org/10.1038/oby.2008.560.

Salbe, A. D., DelParigi, A., Pratley, R. E., Drewnowski, A., \& Tataranni, P. A. (2004). Taste preferences and body weight changes in an obesity-prone population. The American
Journal of Clinical Nutrition, 79, 372-378.

SAS Institute Inc (2013). SAS/ACCESS(r) 9.4 Interface to ADABAS: Reference. Cary, NC: SAS Institute Inc.

Schag, K., Schonleber, J., Teufel, M., Zipfel, S., \& Giel, K. E. (2013). Food-related impulsivity in obesity and binge eating disorder - A systematic review. Obesity Reviews, 14, 477-495. http://dx.doi.org/10.1111/obr.12017.

Stice, E., Yokum, S., Blum, K., \& Bohon, C. (2010). Weight gain is associated with reduced striatal response to palatable food. Journal of Neuroscience, 30, 13105-13109. http:// dx.doi.org/10.1523/JNEUROSCI.2105-10.2010.

Thornton, L. M., Watson, H., Jangmo, A., Welch, E., Wiklund, C., von Hausswolff-Juhlin, Y., ... Bulik, C. M. (2017). Binge-eating disorder in the Swedish National Registers: Somatic comorbidity. International Journal of Eating Disorders, 50, 58-65. http://dx. doi.org/10.1002/eat.22624.

Turner-McGrievy, G., Tate, D. F., Moore, D., \& Popkin, B. (2013). Taking the bitter with the sweet: Relationship of supertasting and sweet preference with metabolic syndrome and dietary intake. Journal of Food Science, 78, S336-S342. http://dx.doi.org/ 10.1111/1750-3841.12008.

U.S. Department of Agriculture \& U.S. Department of Health and Human Services (2010). Dietary guidelines for Americans. Retrieved from https://health.gov/ dietaryguidelines/dga2010/dietaryguidelines2010.pdf.

White, M. A., \& Grilo, C. M. (2005). Psychometric properties of the Food Craving Inventory among obese patients with binge eating disorder. Eating Behaviors, 6 , 239-245. http://dx.doi.org/10.1016/j.eatbeh.2005.01.001.

White, M. A., Whisenhunt, B. L., Williamson, D. A., Greenway, F. L., \& Netemeyer, R. G. (2002). Development and validation of the Food-Craving Inventory. Obesity Research, 10(2), 107-114. http://dx.doi.org/10.1038/oby.2002.17.

World Health Organization (2016). Definition and diagnosis of diabetes mellitus and intermediate hyperglycaemia. Geneva, Switzerland: WHO Document Production Services.

Yanovski, S. (2003). Sugar and fat: Cravings and aversions. The Journal of Nutrition, 133 835S-837S.

Yanovski, S. Z., Leet, M., Yanovski, J. A., Flood, M. N., Gold, P. W., Kissileff, H. R., \& Walsh, B. T. (1992). Food selection and intake of obese women with binge-eating disorder. American Journal of Clinical Nutrition, 56, 975-980. 